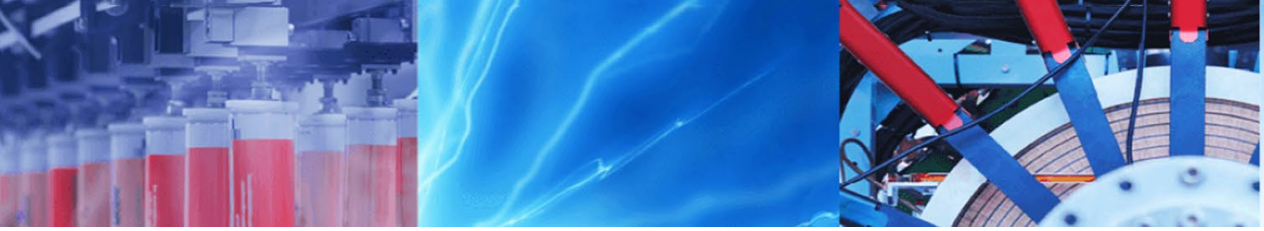

Research Article

\title{
Development of ionic liquid microemulsion for transdermal delivery of a chemotherapeutic agent
}

\author{
Harish Sharma ${ }^{1,3} \cdot$ Gyanesh Kumar Sahu² $\cdot$ Chanchal Deep Kaur ${ }^{3}$ (D)
}

Received: 11 November 2020 / Accepted: 18 January 2021 / Published online: 25 January 2021

(C) The Author(s) 2021 OPEN

\begin{abstract}
Nowadays skin cancers have become a major area of concern because of the continuous exposure to sun rays (UV rays). Hence, the present work focused on the synthesis of an innovative 5-Fluorouracil (5-FU) microemulsion as a topical delivery system mainly used to treat various forms of skin cancer. The topical administration of most of the active compounds is impaired by limited skin permeability due to the presence of skin barriers. In this sequence, the microemulsion represents a cost-effective and convenient drug carrier system that successfully delivers the drug to and across the skin. Unfortunately, 5-FU reveals high toxicity and low tumor affinity became inefficient for patients with the risk of serious side effects. For decreasing of eluding some of its disadvantages we made it more effective by preparing its microemulsion with tween 80 (surfactant), isopropyl alcohol (co-surfactant), oleic acid (oil) in a four-component system. This study emphasized increasing the drug release by multiple times and a topical gel has been formulated and designs to elongate the drug release. All preparation of 5-FU microemulsion was characterized by physicochemical and drug release studies. The size of the 5-FU microemulsion was 550-600 nm confirmed by transmission electron microscopy (TEM) and Zetasizer. The clear microemulsion was prepared at pH 5-6. It shows viscosity in the limit of 13.52-18.23 Pa s. The outcome of the present work is satisfactory for skin cancer treatment.
\end{abstract}

Keywords 5-fluorouracil · Microemulsion · Formulation · Skin cancer · Characterization

\section{Introduction}

Carcinoma is a spoken word that acknowledges the ailment that has the result of unregulated production and partition of cells as a consequence of corpuscular alteration. Skin carcinoma is the unwanted development of dermis units. Dermis carcinoma is in progress when the physique does not restoratively lesion to the deoxyribonucleic acid (DNA) internally dermis units, assent the units to split and proceed uncontrollably [1-3]. Dermis carcinoma can exist as a dusky blur, lesion, bad damage that doesn't recover, or a bump on the skin [4, 5]. Dermis carcinoma is split among two leading parts according to their properties; (a) Non-melanoma dermis carcinoma is a non-spreadable skin cancer it develops in basal dermis cells [6]. These cells are present at the surface of the epidermis. This kind of dermis carcinoma ordinarily arrives in the areas of corporality that the majority disclosed to the sunlight. Most commonly found on the face, nose, forehead, cheeks, lips, ears, scalp, back to the hands, and lower legs. (b) Melanoma; is the rarest form of skin cancer, in which the pigment cell becomes cancerous

\section{Supplementary Information The online version of this article (https://doi.org/10.1007/s42452-021-04235-x) contains supplementary} material, which is available to authorized users.

Chanchal Deep Kaur, drcdkresearch@gmail.com | 'Department of Pharmacy, MJ College, Kohka, Bhilai, Chhattisgarh 490023, India. ${ }^{2}$ Faculty of Pharmaceutical Science, Shri Shankaracharya Group of Institute, Junwani, Bhilai, Chhattisgarh 490020, India. ${ }^{3}$ Shri Rawatpura Sarkar Institute of Pharmacy, Kumhari, Chhattisgarh 493338, India. 
[3]. It repeatedly cultivates on the foot of females and the breastplate, behindhand, skull, and neck of males. However, this kind of dermis carcinoma may for many places on the physique. Melanoma begins in the melanocytes which are the unit in the low most stratum of the epidermis $[7,8]$.

Fluorouracil is an antineoplastic anti-metabolite chemotherapy drug. In-vivo, 5-FU is transformed into the active metabolite 5-fluoroxyuridine monophosphate (F-UMP); substituting uracil, F-UMP assimilates into RNA and DNA thereby hindering there processing, thus preventing cell growth [9]. It has been used in the treatment of numerous solid tumors including colon, rectal, breast, gastric, pancreatic, ovarian, bladder, and liver cancer [10-14]. Its association with low elevations of transient serum aminotransferase during therapy has been implicated in rare cases of clinically apparent acute liver injury. The structure of 5-FU is shown in Fig. S1. FU combines with many enzymes and cofactors like N5-10-methylenetetrahydrofolate, thymidylate synthase, folate cofactor and inhibit covalently bound ternary complex [15-17]. The result of this inhibition of thymidylate convert uracil is important to the reduction of DNA and RNA and causes cancer cell end.

Microemulsions are commonly defined as thermodynamically stable systems; liquid mixtures of oil, water, and surfactant, immiscible or partially miscible are stabilized by added co-surfactants. These are liquid at room temperature carrying negligible vapor pressure [18-20]. Various studies have been done with microemulsions as a drug delivery vehicle because of their many potential and extraordinary benefits [21,22], such as high solubility, lucidity, thermodynamic stability, ease of preparation, high dispersion and absorption rates in comparison with the solvents without the surfactant system [23]. There are numerous properties such as it can be industrialized by a spontaneous emulsification method to improve the solubility and bioavailability of poorly water-soluble drugs, non-toxic and non-irritant hence can be easily applied to the skin and mucous membranes [24]. Therefore, it enhances drug efficiency, with reduced dosage and minimal side effects. At the different ranges of temperatures, they are thermodynamically stable and resign is to a highly small drop size [25]. Therefore, in contrast to other emulsion systems, where the oil droplets may slowly coalesce occur phase separation, in microemulsion preparation phase separation doesn't occur [26]. The transdermal delivery of sparingly soluble drugs is challenging due to of the need for a drug carrier. In the past few decades, ionic liquid (IL)-in-oil microemulsions (IL/O MEs) have been developed as potential carriers. By focusing on biocompatibility, we report on an IL/O ME that is designed to enhance the solubility and transdermal delivery of the sparingly soluble drug, 5 FU.
So, many drugs were used alone or in combination as an anticancer drug [26]. Various work has been so far reported, i.e., formulation of tamoxifen microemulsion with sesame oil and tween 80 as an anticancer drug for oral delivery [27], formulation of surface-active microemulsion for various sparingly soluble drugs to enhance activity towards cancer cell [18], formulation of simvastatin nanoemulsion to treat bone cancer [28] and formulation of 5-FU porous microparticles for liver cancer [29]. However, we did not find a single report on surface modification of 5-FU as microemulsion and implemented for ex-vivo (goat and human cadaver skin) and in-vivo (skin irritation on mice) studies.

The present study reveals the formulation of twentyseven microemulsions by using 5-FU an anticancer drug, which has improved skin permeability and seizing the drug within the shell area thereby drug delivery in a relatively short period. Therefore, the anticancer activity of the particles against melanoma in the skin has been investigated.

\section{Materials and method}

\subsection{Chemicals}

All the reagents were analytical reagent grades. Tween 20, tween 60, tween 80, isopropyl alcohol (IPA), span 80, span 60, ethanol, n-butanol, polyethylene glycol (PEG 400), propylene glycol, isopropyl myristate, oleic acid, castor oil, olive oil, and dimethyl sulphoxide (DMSO) were obtained from Merck, Mumbai, India. 5-FU; a pure drug sample procured from a pharmaceutical company; Neon laboratories Ltd., Mumbai, India as a gift. Triple distilled water was used for microemulsions preparation.

\subsection{Screening of formulation ingredients}

\subsubsection{Screening of oil}

The solubility of 5-FU in various oils; isopropyl myristate, castor oil, olive oil, oleic acid, was determined due to its solubility and tensile efficiency. In brief, the solubility of 5-FU in different oils was determined by the addition of an excess amount of 5-FU in $2 \mathrm{ml}$ of oils by a shaker. For $72 \mathrm{~h}$ the sample was kept to form an equilibrium point. The materials were removed and centrifuged for around $15 \mathrm{~min}$ at 3,000 rpm. Lastly, the supernatant was shifted with the help of a membrane filter to check the percent (\%) of medicament by the help of UV-Visible spectroscopy at $225 \mathrm{~nm}$. 


\section{Screening of surfactants}

The emulsification capacities were checked for three different surfactants namely, tween 20 , tween 60 , and tween 80 , span 60 , span 20 . Firstly prepare $2.5 \mathrm{ml}$ of $15 \%$ surfactant solution next add $4 \mu \mathrm{l}$ oil with continuous stirring to get a clear solution. The emulsions could last $2 \mathrm{~h}$.

\section{Screening of co-surfactants}

During microemulsion preparation, co-surfactant work as a solubilizing agent. Co-surfactant has been combined with surfactant and solubilizes them, to form a clear, transparent solution. Various co-surfactants were investigated such as isopropyl alcohol, ethanol, $n$-butanol, polyethylene glycol, propylene glycol. The titration method was used to determine co-surfactant efficiency [30].

\subsection{Construction of phase diagram}

The phase diagram displays the concentration of surfactant, co-surfactant, oil, and water for the preparation of microemulsion. Pseudo ternary phase diagrams have been also constructed to examine the influence on the region of microemulsion existence of the surfactant to the cosurfactant ratio $(\mathrm{S} / \mathrm{CoS})$. To achieve the best one microemulsion, each diagram has a precise weight ratio of Smix and oil mixtures to prepare a total of twenty-seven formulation for different ratios, i.e., 1:1, 2:1, 3:1. The mixtures of surfactant, co-surfactant (Smix), and oil combination were mixed slowly with continuous stirring. The quantity of water depending on the mixtures was assessed visually like microemulsion. The phase diagram for the preparation of microemulsion was optimized by the 'triangular graph constructor' software. The general ternary-phase diagram of microemulsion represents in Fig. 1a (supporting document; Fig. S2, S3, and S4). Table S1, S2, and S3 represent the percentage of surfactant, co-surfactant, water, and oil at 1:2, 2:1 \& 3:1 ratio of tween 80:IPA. Table S4, shows the formulation of all three (ME1, ME2, and ME3) 5-FU microemulsions (5\%).

\subsection{Characterization}

After getting the best formulation for 5-FU microemulsions, it was further studied for its characteristics such as visual inspection, thermodynamic stability which include stress test and centrifugation. The zeta potential
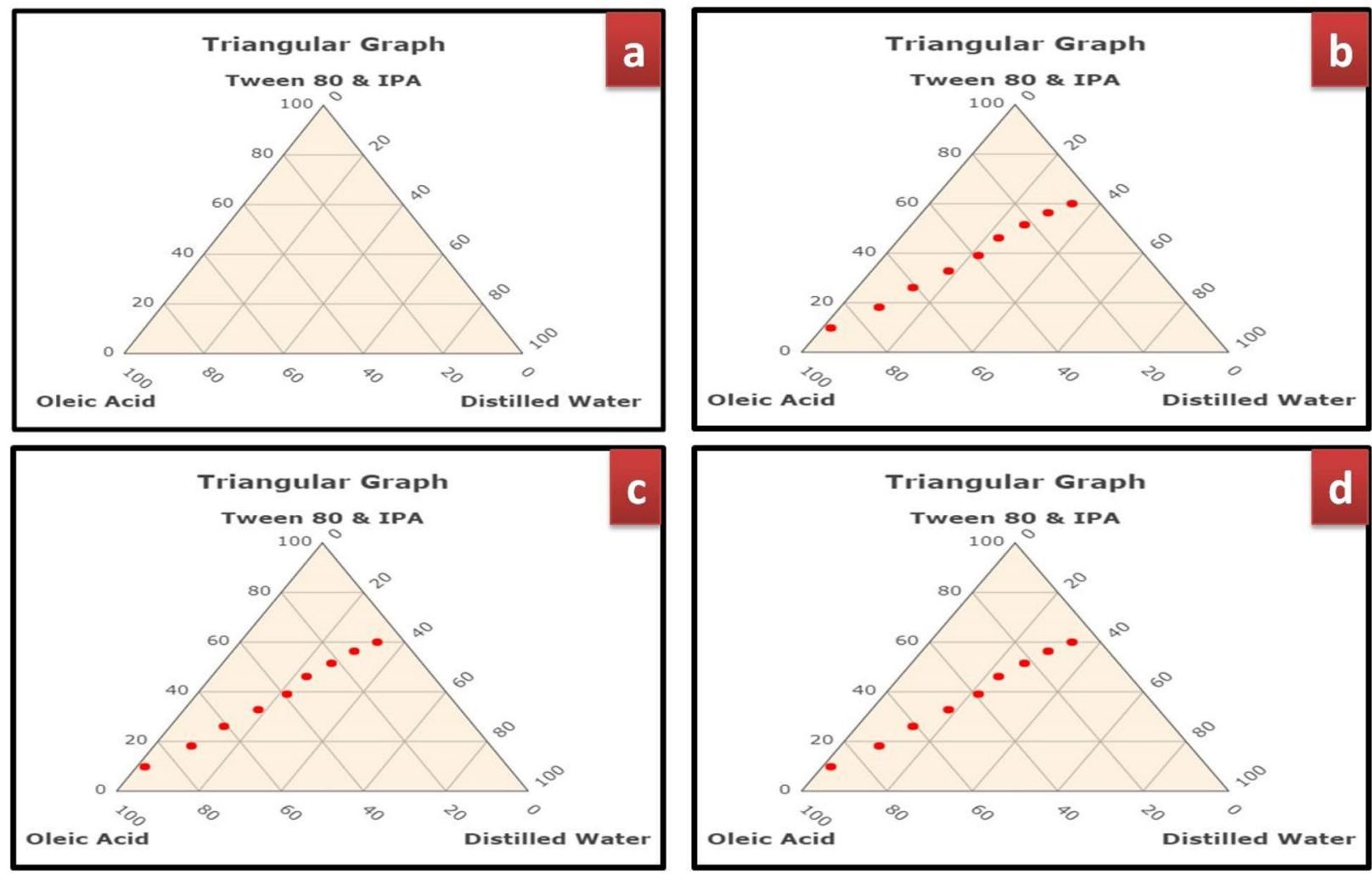

Fig.1 Pseudo ternary phase diagram of 5-FU microemulsions; phase diagram representation (a); 1:1 weight ratio (b); 2:1 weight ratio (c); 3:1 weight ratio (d) 
of microemulsions samples was determined using a Zetasizer 2000 (Malvern Co., Worcestershire, UK). Transmission electron microscopy JEOL JEM-1010 (Jeol, Tokyo, Japan) operating at an accelerating voltage of $80 \mathrm{kV}$ was used to determine the size and morphology of the 5-FU microemulsion. A Systronic $\mathrm{pH}$-meter was used for measuring the $\mathrm{pH}$ of the prepared microemulsion. While viscosity was measure by Brook field viscometer model WDV-8.

\subsubsection{Spreadability study}

The spreadability apparatus consists of a wooden block with marking and two glass slides had a pan mounted on a pulley. The required amount of microemulsion kept between two glass slides and $100 \mathrm{~g}$ weight was placed on the upper glass slide for 2 min to obtain a uniform thickness of the formulation. Measurement of spreadability was taken as the time(s) required separating the two slides.

The spreadability was calculated by using the following equation:

$S=(m \times I) / t$

where, $S$ is spreadability; $m$ is the weight placed on the upper slides; I is the length of the glass slide and; $t$ is the time taken in seconds.

\subsubsection{Ex-vivo drug permeation studies}

Ex-vivo drug permeation studies (goat and human cadaver skin), were performed in vertical Franz diffusion cells that have a diffusion surface of $0.63 \mathrm{~cm}^{2}$ (Logan Instruments, Somerset, NJ) at room temperature [30].

Goatskin Goatskin, weighing 8-10 g, were obtained from the local market at Bhilai, Chhattisgarh, India. Polypropylene cages were further used to store them. Ethical approval and the guidelines to perform the animal studies were obtained from the Institutional Animal Ethics Committee (IAE), Shri Rawatpura Sarkar Institute of Pharmacy, Raipur, India that was strictly followed throughout the studies.

Human cadaver skin Franz diffusion cell was applied in the test for ex-vivo diffusion of medicament with a volume of $6 \mathrm{~cm}$ was used. It has two chambers; the upper receptor chamber was covered with a cellulose acetate membrane with the addition of the release media. The next chamber is a donor chamber that was mounted on the membrane. $1 \mathrm{~g}$ samples of the microemulsion were loaded into the donor chambers with stirring at $800 \mathrm{rpm}$. Formulation from the receptor chamber is taken at a frequent interval of times throughout $3 \mathrm{hr}$ and the concentration of the medicament was determined by UV-Visible spectrophotometric method using the standard curve.
The quantity of medicament distributed at many time intervals was calculated and the graph plotted against time.

\subsubsection{Skin sensitivity studies}

The cytotoxicity test was done in A431 cells, at National centre for Cell Sciences, Department of Biotechnology, Pune, (M.H.), using the colorimetric method to determine skin irritation. In brief, before one week of the experiment, mice were adapted to laboratory conditions. The humidity of the room was maintained up to $40-45 \% \mathrm{RH}$ and the temperature was monitored at $25^{\circ} \mathrm{C}$. The hair on the dorsal side of the mice was carefully removed with an electric clipper in the direction of the tail to the head (approx. $5 \mathrm{~cm}$ ) without damaging the skin. Now, animals were divided into three groups $(n=9)$ and treated as follows: group I: negative controlled (no treatment), group II: test formulation, group III: applied formalin a standard irritant $(0.8 \% \mathrm{v} / \mathrm{v})$. Test formulations $(0.5 \mathrm{~g})$ were applied uniformly on the dorsal region by uniform spreading within the area for continuous 7 days. The treated skin was inspected visually for erythema and edema. The mean erythemal and edema scores were recorded depending on the degree of erythema.

\section{Results}

\subsection{Solubility studies}

To prepare a microemulsion, initially, the solubility of oil, surfactant, and co-surfactant was determined. For that firstly, the screening of different oil, i.e., isopropyl myristate, oleic acid, castor oil, and olive oil was done [31]. Table 1, depicts the solubility of 5-Fluorouracil in different oil and concluded oleic acid was best to prepare microemulsion with the highest solubility along with the solubility of oil, surfactants, co-surfactants respectively. The results indicate an enhanced solubility of 5-FU in the optimized formulation when microemulsion was compared to its respective individual ingredients.

Based on the screening of all reagents, optimized constituents to prepare 5-FU microemulsion (5\%) were obtained (Table S4).

The increased solubility of 5-FU in 3:1 surfactant to cosurfactant ratio can be attributed to solute-solvent interactions such as hydrogen bonds, Van der Waals forces, and $\pi-\pi$ interactions occurring between aromatic rings of imidazolium cation and 5-FU. 
Table 1 Solubility of 5-Fluorouracil in different oil, surfactant, and co-surfactant at room temperature

\begin{tabular}{lllllc}
\hline Oil & Solubility $(\mathrm{mg} / \mathrm{ml})$ & Surfactant & Solubility $(\mathrm{mg} / \mathrm{ml})$ & Co-surfactant & Solubility $(\mathrm{mg} / \mathrm{ml})$ \\
\hline Isopropyl myristate & $165.28 \pm 0.23$ & Tween 80 & $105.28 \pm 0.22$ & Isopropyl alcohol & $107.28 \pm 0.25$ \\
Oleic acid & $183.12 \pm 0.21$ & Tween 60 & $100.45 \pm 0.24$ & Ethanol & $43.12 \pm 0.21$ \\
Castor oil & $44.26 \pm 0.25$ & Tween 20 & $93.12 \pm 0.21$ & N-Butanol & $58.27 \pm 0.19$ \\
Olive oil & $32.61 \pm 0.19$ & Span 80 & $58.27 \pm 0.19$ & Polyethylene glycol & $69.62 \pm 0.24$ \\
- & - & Span 20 & $31.62 \pm 0.22$ & Propylene glycol & $79.62 \pm 0.24$ \\
\hline
\end{tabular}

\subsection{Pseudo-ternary phase diagram and preparation of MEs}

The microemulsion formulation is a complex mixture of oil, surfactant, cosurfactant, water, and drug and the majority of its properties are dependent upon the droplet size of the internal phase [32,33]. Figure $1 b, c$, d reveals a pseudo-ternary phase diagram of 5-FU microemulsion with Tween 80, IPA, oleic acid, and water at room temperature. The "water dilution lines" were drawn, reflecting an increase in water content and a decrease in Smix levels. The water was steadily titrated with a Smix and visually observed in the device for clarity or turbidity. The mixture was stirred over the vortex mixer during every addition of water. Since, after stirring, a clear and transparent mixture was collected, the sample was considered monophasic. As a point in the phase diagram, every composition of the monophasic emulsion was marked. It was assumed that the area protected by these points was the microemulsion region of life. In phase diagrams, the transparent area of the microemulsion life zone is presented. Based on visual observation, the remainder of the area on the phase diagram represents the biphasic, turbid, and traditional emulsions. The total area under the microemulsion zone was inversely proportional to the ratio of $\mathrm{S}_{\text {mix }}$. The area of the microemulsion zone became enlarged as the $S_{\text {mix }}$ ratio decreased, reaching the maximum point at $S_{\text {mix }}$ of 3:1. The ternary phase study reveals that the extreme amount of water has engulfed in the microemulsion system when the $S / C o$ ratio was $3: 1$. Increased quantity of water and decreased amount of surfactant-co-surfactant and oil provides a larger opportunity for the solubilization of 5-FU which is an added advantage for less viscous and transparent microemulsion.

\subsection{Transmission electron microscopy (TEM)}

The shape and size of the as-prepared 5-FU microemulsion were determined by transmission electron microscopy (TEM) [34]. Surface morphology showed an oval shape with $560 \mathrm{~nm}$ size. Solubility is also depending on the size of the particles, as the size decreases the solubility of microemulsions increases. Hence TEM image confirms the micro size, oval shape, and uniform distribution of the 5-FU (Fig. 2a, b).

\subsection{Measurement of particle size and distribution}

The size of the microemulsion globule was determined by Zetasizer. The average globule size was $510 \mathrm{~nm}$. The droplet size was calculated using the Stokes-Einstein relationship by Zetasizer Software. Figure 3, shows three
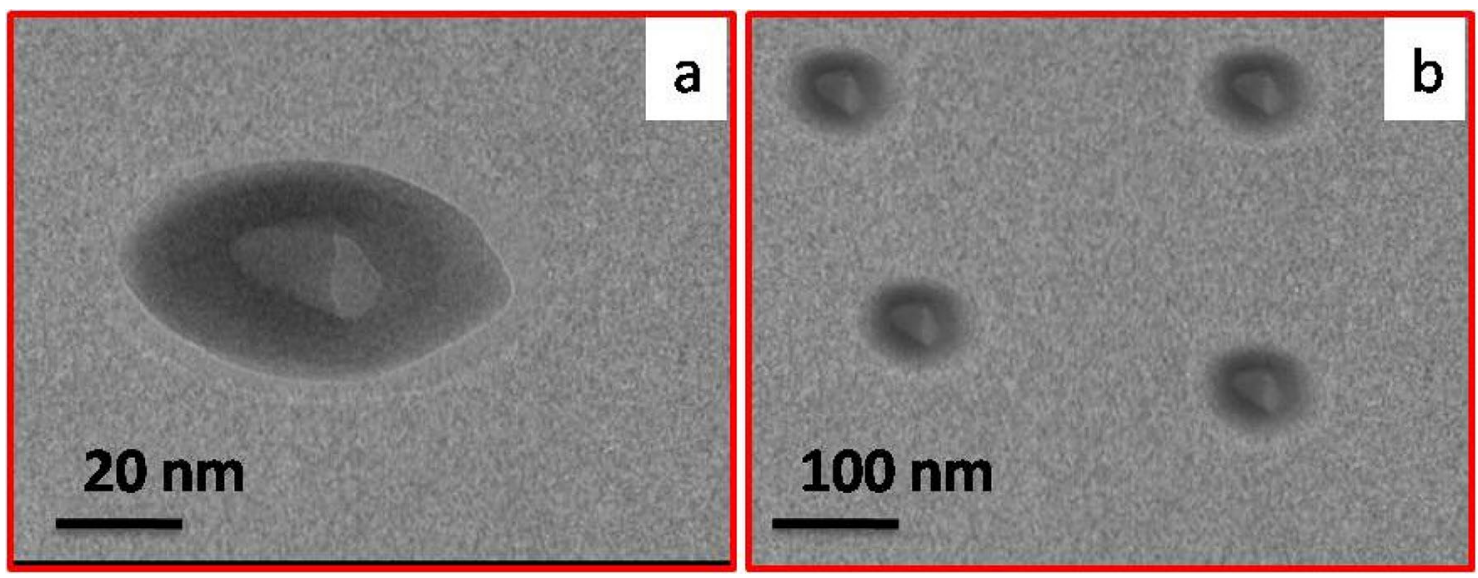

Fig. 2 Transmission electron microscopy (TEM) image of the 5-FU microemulsion (3:1) 


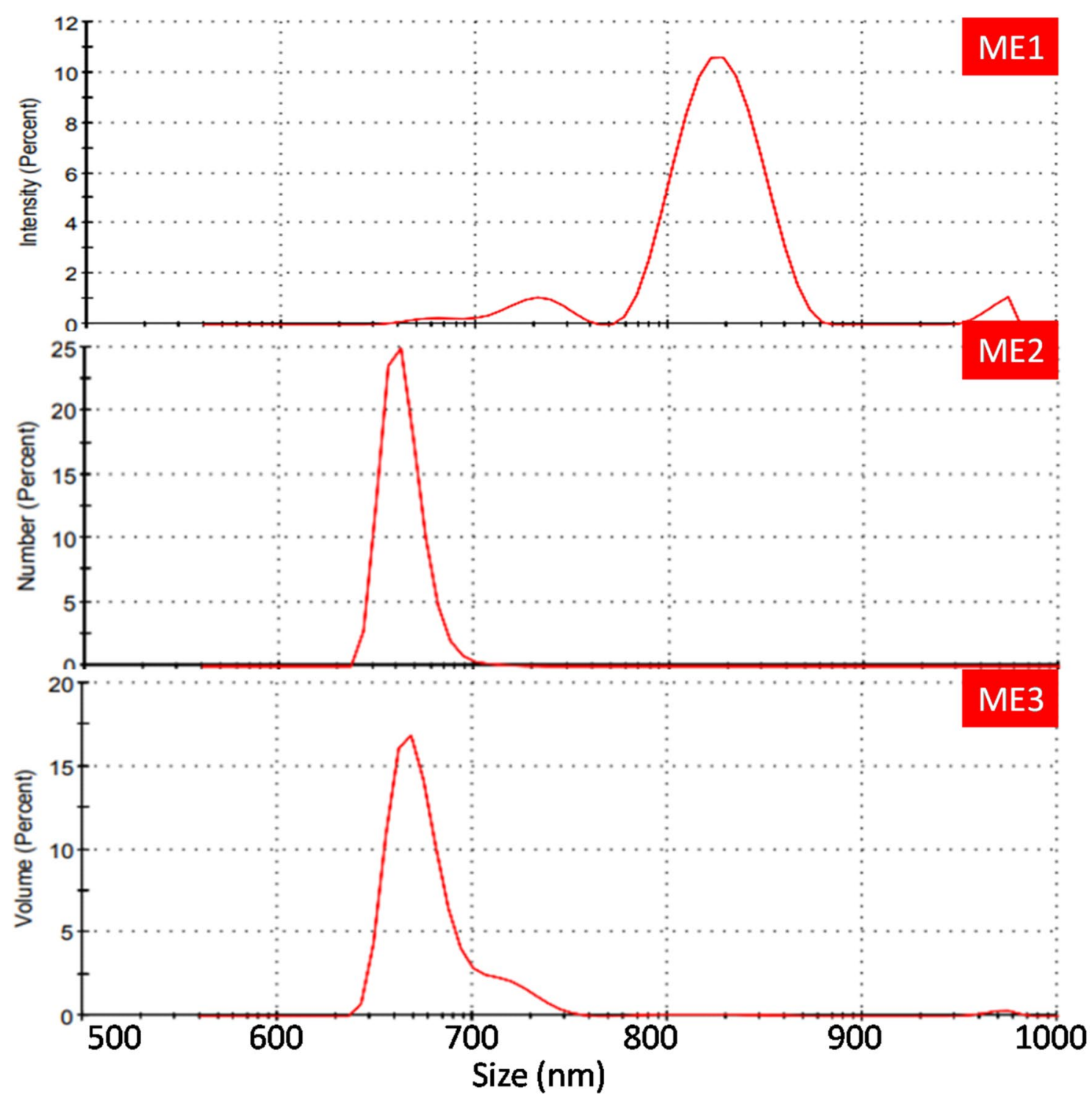

Fig. 3 Zeta potential micrograph of 5-FU microemulsion

peaks and the most intense peak obtained at $689.2 \mathrm{~nm}$. Thus, obtained results show homogeneous distribution of microemulsion globules and it is in the required range, consequently, a translucent microemulsion is formulated efficaciously. Aggregations will not take place due to the slightly negative charge of the droplets [35].

\subsection{Viscosity and pH measurements}

As viscosity is the friction between the inter molecules in the microemulsion, it shows the thickness of the prepared material. Brookfield Viscometer was used to study the viscosity of the as-prepared microemulsion formulation. The viscosity of ME3 was found to be $18.23 \pm 0.22 \mathrm{~Pa}-\mathrm{s}$ as shown in Table S5. Due to its highest viscosity, ME3 was selected for topical application.

The $\mathrm{pH}$ of the prepared 5-FU was determined to know about the $\mathrm{pH}$ of the best microemulsion formulation with the help of a pH meter. Figure 4 represents the $\mathrm{pH}$ values of microemulsions batches. Almost all microemulsions show $\mathrm{pH}$ at 5.64-5.9, approximating the usual transdermal fluid $\mathrm{pH}$ range, which is among the elements of formulation that can significantly lower the irritation created after implementation [36].

\subsection{Thermodynamic stability studies}

Thermodynamic stability reveals the stability of prepared drug microemulsion in terms of different temperatures, 


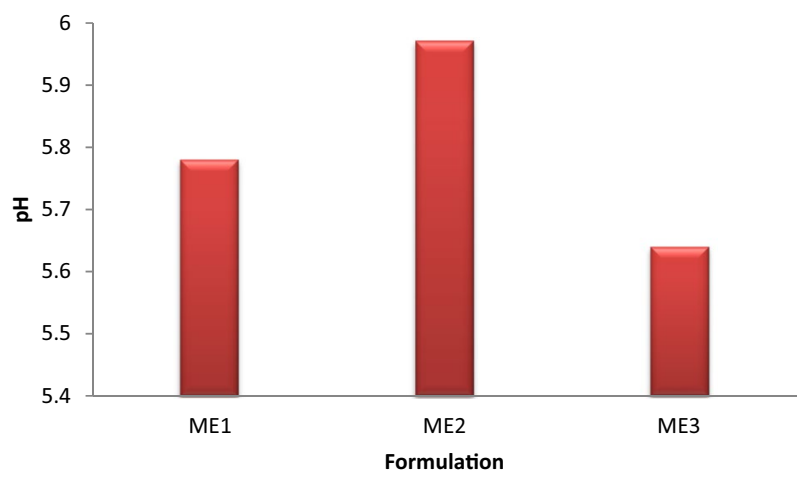

Fig.4 pH values of microemulsions batches

Table 2 Spreadability of prepared microemulsion batches

\begin{tabular}{lll}
\hline S. No & Batch No & $\begin{array}{l}\text { Spreadabil- } \\
\text { ity (g.cm/ } \\
\text { sec) }\end{array}$ \\
\hline 1 & ME1 & 5.14 \\
2 & ME2 & 4.82 \\
3 & ME3 & 4.22 \\
\hline
\end{tabular}

energy, or the equilibrium state of the prepared drug microemulsions with the environment [37]. To determine the thermodynamic stability of the 5-FU microemulsions, firstly placed all these three as prepared ME1, ME2, and ME 3 at different temperatures, i.e., $2-4{ }^{\circ} \mathrm{C}, 20-25^{\circ} \mathrm{C}$, $35-50^{\circ} \mathrm{C}$ for 3 months and observed there were no phase separation and color change occurred during this period. Next, subject these formulations to centrifugations at $1000 \mathrm{rpm}$ and $3000 \mathrm{rpm}$ for $1 \mathrm{~h}$ (Table S6, Fig S5.). Centrifugation can accelerate the rate of creaming or sedimentation which demonstrates that the breakdown of an emulsion can be related to the action of gravitational force. Centrifugation was done to evaluate for phase separation, cracking, or coalescence, and again no changes were observed in the microemulsion.

\subsection{Spreadability study}

Spreadability measurement of prepared microemulsion batches was done by wooden block with scale and two glass slides. ME3 shows $4.22 \mathrm{~g} . \mathrm{cm} / \mathrm{s}$ spreadability on skin (Table 2).

\subsection{Ex-vivo studies}

The ex-vivo studies were done on both goatskin and human cadaver skin by the use of Franz diffusion cell, to know the efficacy of the 5-FU microemulsion formulation for permeation on skin. The below figure shows the efficiency of different 5-FU microemulsions (ME1, ME2, and ME3) with free 5-FU in goat and human cadaver skin. It was observed that 5-FU with ME3 formulation for goatskin showed $86 \%$ permeation compared to other ratios ME2 (78\%), ME1 (53\%), and free 5-FU (25\%). The increase in the percentage of permeation is due to microemulsion formation in free 5-FU, which enhances its surface area. For human cadaver skin permeability results of 5-FU obtained almost the same pattern of results that ME3 shows the best skin permeability with no side effect on the skin. In general, when 5-FU is used alone on the upper layer of the skin the surface was very less and can easily penetrate the inner layer of the skin. Figure $5 \mathrm{a}$, shows the comparative skin retention profile for all the formulation 5-FU ME with free 5-FU. The value of skin retention for free 5-FU was found to be around $32 \%$. However, microemulsion of 5-FU shows better results,
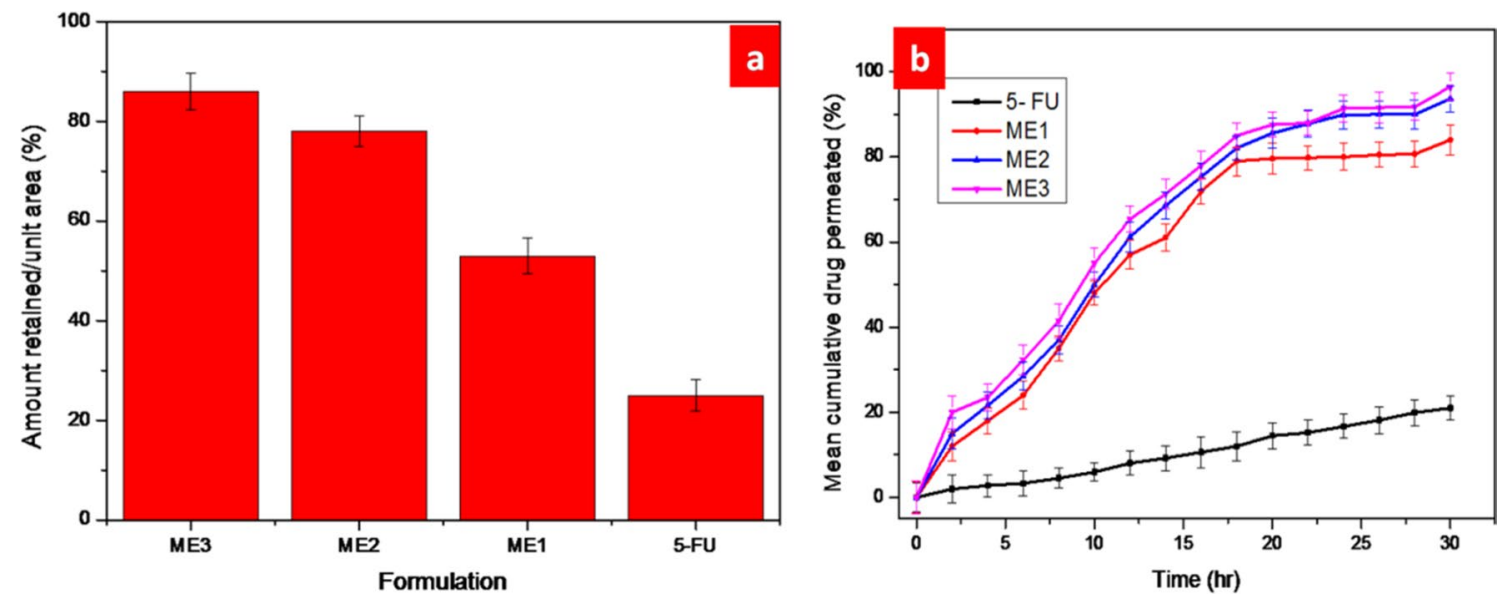

Fig. 5 Comparative skin retention profile for all the formulation 5-FU ME with free 5-FU (a); Percentage (\%) mean cumulative 5-FU microemulsions penetration in the skin (b) 
i.e., ME1, ME2, and ME3 are $53 \%, 78 \%$, and $86 \%$ respectively. Thus, based on the above results, it was concluded that microemulsion based drug provides better drug deposition on skin compare to free 5-FU (Fig. 5b).

\subsection{Cytotoxicity test (MTT assay)}

MTT assay the high-throughput cell-based assay was used to evaluate the cytotoxic response of different cell lines to different oncology products. MTT assay is a test done in the lab and a usual colorimetric assay (an assay that measures color changes) for determining growth in a cell. In this paper, the MTT assay was reformed as a chemosensitivity test, and its potential was examined. This technique also has numerous benefits regarding speed, quantitation, managing of various samples, and cell number is essential for the assay. The application of this assay to chemosensitivity testing appears to be valuable and advantageous.

MTT examines cell respiration and the extent of formazan made is proportionate to the number of living cells existing in the culture. An escalation or reduction in cell number results in a concomitant change in the quantity of formazan made, demonstrating the degree of cytotoxicity initiated by the drug. $\mathrm{IC}_{50}$ (concentration) of the tested drug capable of causing the death of $50 \%$ of the cells and can predict the degree of its cytotoxic effect. The lower the value, the more cytotoxic the substance will be. Figure 6 , reveals the comparative graph of the $\mathrm{IC}_{50}$ of certain chemotherapeutic drugs against human cancer cell lines.

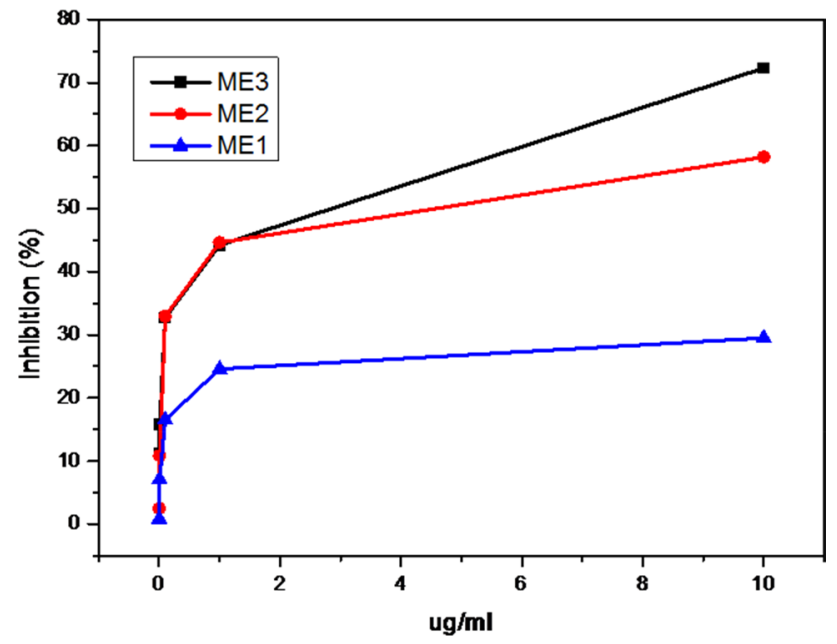

Fig. 6 MTT assay to determine the $\mathrm{IC}_{50}$ and analyze their effect on cell viability in 5-FU microemulsion (ME1, ME2, and ME3)

\subsubsection{Skin irritation studies}

ME1, ME2, and ME3 samples formulated show zero erythema and edema score index for negative and testcontrolled values. The microscopic appearances of the controlled mice skin sections (without treatment) and the sections of skin treated with all formulation shown in Fig.S6. The Draize score for skin irritation for negative and test controlled were zero (no effect) while positive control for erythema was $1.11 \pm 0.03$ with edema $0.89 \pm 0.014$. Anatomical and pathological changes established in this study did not provide the safety of tested formulations on mice skin.

\section{Conclusion}

Topical 5-FU is approved for the treatment of superficial basal cell carcinoma and actinic keratosis. However, 5-FU suffers from poor skin permeation. The above formulations have been successfully applied to improve the skin permeability of small and large molecules, and even microparticles, by creating micron-sized pores in the stratum corneum layer of the skin.

In summary, successfully prepared a more efficient 5-FU microemulsion via the phase titration method. Later on, implemented as a carrier system for topical delivery on dermis carcinoma with improved solubility and permeability. 5-FU was characterized by physicochemical evaluation and shows particle size in the limit of $500-600 \mathrm{~nm}$ at $\mathrm{pH} 5.64-5.97$. Around $95.57-83.67 \%$ quantity of 5-FU was released from all ME. To achieve effective results different ratios of microemulsions were prepared and implemented on Goat and human cadaver skin and found ME3 was successful in treating dermal carcinoma for 3 months as compared to ME1 and ME2. Ex-vivo permeation studies reveal a great increment in flux as well as an increasing amount of drug-infused in $24 \mathrm{~h}$ using ME solution. Skin irritation is also very low as compared to free 5 -FU. Thus this work will expose the novel method for further research as a probable drug delivery system with excellent stability and release profile. All the other formulations (ME1 and ME2) were also found to be equally good in their physicochemical characteristic.

Acknowledgement The authors would like to thank you for the drug as a gift sample from Neon Laboratories Ltd., Mumbai, India. We also acknowledge Shri Shankaracharya Group of Institute, Junwani, Bhilai and Shri Rawatpura Sarkar Institute of Pharmacy, Raipur, Chhattisgarh India for providing basic lab facilities. And we also thank the Indian Institute of Integrative Medicine, Jammu, All India Medical Institute of Medical Sciences (AlIMS) New Delhi, Malvern Instruments Ltd., Deshpandey laboratory India for microemulsion analysis. 
Author contributions Harish Sharma- Material preparation, data analysis, draft preparation. Gyanesh Kumar- Commented on previous version of manuscript. Chanchal Deep Kaur- Supervision and finalization.

Code availability Not applicable.

Availability of data and material Not applicable.

\section{Compliance with ethical standards}

Conflicts of interest Authors declare no competing financial and non-financial interest and.

Open Access This article is licensed under a Creative Commons Attribution 4.0 International License, which permits use, sharing, adaptation, distribution and reproduction in any medium or format, as long as you give appropriate credit to the original author(s) and the source, provide a link to the Creative Commons licence, and indicate if changes were made. The images or other third party material in this article are included in the article's Creative Commons licence, unless indicated otherwise in a credit line to the material. If material is not included in the article's Creative Commons licence and your intended use is not permitted by statutory regulation or exceeds the permitted use, you will need to obtain permission directly from the copyright holder. To view a copy of this licence, visit http://creativecommons .org/licenses/by/4.0/.

\section{References}

1. Freeman RG, Knox JM, Heaton CL (1964) The treatment of skin cancer. A statistical study of 1,341 skin tumors comparing results obtained with irradiation, surgery, and curettage followed by electrodesiccation. Cancer 17(4):535-538

2. Scholz A (1997) History of skin cancer. In: Altmeyer P, Hoffmann $\mathrm{K}$, Stücker M (eds) Skin cancer and UV radiation. Springer, Berlin

3. Recio A (2019) Tumour growth activation by the central nervous system-An integrative theory of cancer. Stress Health 35(4):569-581

4. Jones OT, Ranmuthu CKI, Hall PN, Funston G, Walter FM (2020) Recognising skin cancer in primary care. Adv Ther 37(1):603-616

5. Migden M (2020) Basal cell carcinoma - Advances in treatment and research. https://www.springer.com/gp/book/9783030268 862. Accessed from 2020

6. Physical assessment for nurses and healthcare Professionals, 3rd Edition | Wiley [Internet]. Wiley.com. [cited 2020 Dec 24]. https://www.wiley.com/en-in/Physical+Assessment+for+Nurse s+and+Healthcare+Professionals $\% 2 C+3 r d+$ Edition-p-97811 19108993. Accessed from 24 Dec 2020

7. Baumann BC, MacArthur KM, Brewer JD, Mendenhall WM, Barker CA, Etzkorn JR et al (2020) Management of primary skin cancer during a pandemic: multidisciplinary recommendations. Cancer 126(17):3900-3906

8. Maiti A, Chatterjee B (2020) Improving detection of melanoma and naevus with deep neural networks. Multimed Tools Appl 79(21):15635-15654

9. Kligman AM, Levine N, Romine KA (1997) The short-term use of 5-Fluorouracil to identify subclinical actinic keratoses in young adults with subsequent long-term application of tretinoin to prevent tumor progression. In: Altmeyer P, Hoffmann $\mathrm{K}$, Stücker M (eds) Skin cancer and UV radiation. Berlin, Heidelberg, Springer, pp 1273-1283
10. Vokes EE, Panje WR, Weichselbaum RR (1991) 5-Fluorouracil modulation in head and neck cancer. In: Banzet $P$, Holland JF, Khayat D, Weil M (Eds.). Proceedings of the 3rd international congress on neo-adjuvant chemotherapy. Paris: Springer; $\mathrm{p}$. 54-7

11. Mayer M, Linder M, SchremI W, Schlag P, Queißer WA (1979) Controlled prospective study of adjuvant 5-Fluorouracil and BCNU therapy in stomach carcinoma. In: Herfarth $\mathrm{CH}$, Schlag PM (eds) Gastric cancer. Berlin, Heidelberg, Springer p, pp 357-360

12. Popiela T, Zembala M, Oszacki J, Jedrychowski W (1982) A follow-up study on chemoimmunotherapy (5-fluorouracil and BCG) in advanced gastric cancer. Cancer Immunol Immunother 13(3):182-184

13. Van Ruth S, Jansman FGA, Sanders CJ (2006) Total body topical 5 -fluorouracil for extensive non-melanoma skin cancer. Pharm World Sci 28(3):159

14. Longley DB, Harkin DP, Johnston PG (2003) 5-Fluorouracil: mechanisms of action and clinical strategies. Nat Rev Cancer 3(5):330-338

15. Aschele C, Sobrero A, Guglielmi A, Mori A, Tixi L, Bolli E et al (1994) 5-Fluorouracil may have different mechanisms of action depending on the dosing schedule: clinical implications. In: Banzet P, Holland JF, Khayat D, Weil M (eds) Cancer treatment an update. Springer, Paris, pp 804-808

16. Morere JF, Duran A, Tcherakian F, Boaziz C, Battesti JP, Israel L et al (1994) Cisplatinum and 5-Fluorouracil in small cell lung cancer. In: Banzet P, Holland JF, Khayat D, Weil M (eds) Cancer treatment an update. Springer, Paris, pp 328-330

17. Saif MW, Makrilia N, Syrigos K (2020) CoFactor: Folate requirement for optimization of 5-Fluorouracil activity in anticancer chemotherapy [Internet]. Vol. 2010, J Oncol. https://www. hindawi.com/journals/jo/2010/934359/. Accessed from 2010

18. Ali MdK, Moshikur RM, Wakabayashi R, Moniruzzaman M, Kamiya N, Goto M (2020) Biocompatible ionic liquid surfactant-based microemulsion as a potential carrier for sparingly soluble drugs. ACS Sustain Chem Eng 8(16):6263-6272

19. Bhalke RD, Kulkarni SS, Kendre PN, Pande VV, Giri MA (2020) A facile approach to fabrication and characterization of novel herbal micro emulsion-based UV shielding cream. Future J Pharm Sci 6(1):76

20. Tokuda H, Tsuzuki S, Susan MdABH, Hayamizu K, Watanabe $M$ (2006) How ionic are room-temperature ionic liquids? An indicator of the physicochemical properties. J Phys Chem B 110(39):19593-19600

21. Goindi S, Narula M, Kalra A (2016) Microemulsion-based topical hydrogels of tenoxicam for treatment of arthritis. AAPS PharmSciTech 17(3):597-606

22. Senapati S, Mahanta AK, Kumar S, Maiti P (2018) Controlled drug delivery vehicles for cancer treatment and their performance. Signal Transduct Target Ther 3(1):1-19

23. Mishra A, Panola R, Rana AC (2014) Microemulsions: as drug delivery system. J Sci Innov Res 3(4):467-474

24. Chen J, Ma X, Yao G, Zhang W, Zhao Y (2018) Microemulsionbased anthocyanin systems: effect of surfactants, cosurfactants, and its stability. Int J Food Prop 21(1):1152-1165

25. Salimi A, Panahi-Bazaz M-R, Panahi-Bazaz E (2020) A novel microemulsion system for ocular delivery of azithromycin: Design, characterization and ex-vivo rabbit corneal permeability. J Nat Pharma Prod https://sites.kowsarpub.com/jjnpp /articles/13938.html\#abstract. Accessed from 2020

26. Liu Y, Li Q, Zhou L, Xie N, Nice EC, Zhang H et al (2016) Cancer drug resistance: redox resetting renders away. Oncotarget 7(27):42740-42761

27. Dehghani F, Farhadian N, Golmohammadzadeh S, Biriaee A, Ebrahimi M, Karimi M (2017) Preparation, characterization and 
in-vivo evaluation of microemulsions containing tamoxifen citrate anti-cancer drug. Eur J Pharm Sci 96:479-489

28. Tao S, Chen S, Zhou W, Yu F, Bao L, Qiu G et al (2020) A novel biocompatible, simvastatin-loaded, bone-targeting lipid nanocarrier for treating osteoporosis more effectively. RSC Adv 10(35):20445-20459

29. Wen Y, Liu Y, Zhang H, Zou M, Yan D, Chen D et al (2019) A responsive porous hydrogel particle-based delivery system for oncotherapy. Nanoscale 11(6):2687-2693

30. Bao Q, Newman B, Wang Y, Choi S, Burgess DJ (2018) In vitro and ex vivo correlation of drug release from ophthalmic ointments. J Control Release 276:93-101

31. Patel V, Kukadiya H, Mashru R, Surti N, Mandal S (2010) Development of microemulsion for solubility enhancement of clopidogrel. Iran J Pharm Res IJPR 9(4):327-334

32. Moghimipour E, Salimi A, Leis F (2012) Preparation and evaluation of tretinoin microemulsion based on pseudo-ternary phase diagram. Adv Pharm Bull 2(2):141-147

33. Chouhan P, Saini TR (2016) D-optimal design and development of microemulsion based transungual drug delivery formulation of ciclopirox olamine for treatment of onychomycosis. Indian J Pharm Sci 78(4):498-511
34. Shen L-N, Zhang Y-T, Wang Q, Xu L, Feng N (2014) Preparation and evaluation of microemulsion-based transdermal delivery of total flavone of rhizoma arisaematis. Int J Nanomedicine 9:3453-3464

35. Patel RB, Patel MR, Bhatt KK, Patel BG (2013) Formulation consideration and characterization of microemulsion drug delivery system for transnasal administration of carbamazepine. Bull Fac Pharm Cairo Univ 51(2):243-253

36. Hathout RM, Woodman TJ, Mansour S, Mortada ND, Geneidi AS, Guy RH (2010) Microemulsion formulations for the transdermal delivery of testosterone. Eur J Pharm Sci Off J Eur Fed Pharm Sci 40(3):188-196

37. Maghraby GME (2012) Microemulsions as transdermal drug delivery systems. Curr Nanosci. Vol. 8. pp 504-511. https://www. eurekaselect.com/100595/article. Accessed from 2012

Publisher's Note Springer Nature remains neutral with regard to jurisdictional claims in published maps and institutional affiliations. 